\title{
The role of blood loss and diet in the aetiology of mild iron deficiency in premenopausal adult New Zealand women
}

\author{
Anne-Louise M Heath ${ }^{1}$, C Murray Skeaff ${ }^{1}$, Sheila Williams ${ }^{2}$ and Rosalind S Gibson ${ }^{1, *}$ \\ 'Department of Human Nutrition, University of Otago, PO Box 56, Dunedin, New Zealand: ${ }^{2}$ Department of \\ Preventive \& Social Medicine, University of Otago, PO Box 56, Dunedin, New Zealand
}

Submitted 2 November 1999: Accepted 17 February 2000

\begin{abstract}
Objective: To investigate the role of blood loss and diet in the aetiology of mild iron deficiency (MID) in premenopausal New Zealand women. Mild iron deficiency was defined as low, but not necessarily exhausted, iron stores (i.e. serum ferritin $<20 \mu \mathrm{g} /$ $\mathrm{L}$ ) in the absence of anaemia (i.e. haemoglobin $\geq 120 \mathrm{~g} / \mathrm{L}$ ).

Design: Cross-sectional study of a volunteer sample of premenopausal adult women. Information on habitual dietary intakes (using a specially designed and validated computerised iron food frequency questionnaire), health and demographic status, sources of blood loss (including menstrual blood loss estimated using a validated menstrual recall method), contraceptive use, height and weight, haemoglobin, serum ferritin and C-reactive protein were collected.

Setting: Dunedin, New Zealand during 1996/1997.

Participants: Three hundred and eighty-four women aged 18-40 years.

Results: The characteristics that were associated with an increased risk of MID were: low meat/fish/poultry intake, high menstrual blood loss, recent blood donation, nose bleeds, and low body mass index. The protective factors included shorter duration of menstrual bleeding, and multivitamin-mineral supplement use in the past year.

Conclusions: There are a number of potentially modifiable factors that appear to influence risk of MID. Women with low menstrual blood loss may be able to decrease their risk of MID by increasing their meat/fish/poultry intake, while those with a higher menstrual blood loss may be able to decrease their risk by decreasing their menstrual blood loss, perhaps by changing their method of contraception. Women should be encouraged to maintain a healthy body weight, and those who choose to donate blood, or who experience nose bleeds, should have their iron stores monitored.
\end{abstract}

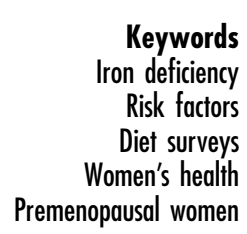

Keywords on deficiency

Risk factors

Women's health

Premenopausal women
There is a growing interest in the presence of iron deficiency without any clinical or biochemical evidence of anaemia, and the extent to which it may be associated with adverse health consequences. Some reports have suggested that iron deficiency, without anaemia, may result in impaired cognitive function ${ }^{1}$ and decreased work performance $e^{2,3}$.

The early stages of iron deficiency are characterised by a gradual decline in the amount of iron stored in the liver, until eventually stores become completely exhausted. These changes are reflected by a progressive fall in serum ferritin from between 20 and $12 \mu \mathrm{g} / \mathrm{L}$, to less than $12 \mu \mathrm{g} / \mathrm{L}$, at which point the stores are considered to be exhausted. In this study of premenopausal women, we have defined mild iron deficiency (MID) as the existence of low but not necessarily exhausted iron stores (i.e. serum ferritin $<20 \mu \mathrm{g} / \mathrm{L}$ ), in the absence of anaemia (i.e. haemoglobin $\geq 120 \mathrm{~g} / \mathrm{L}$ ).

Women of child-bearing age are known to be more vulnerable to mild iron deficiency than post-menopausal women. In a recent national survey in the United Kingdom $^{4}$ approximately $40 \%$ of women aged 18 to 40 years had a serum ferritin level of less than $25 \mu \mathrm{g} / \mathrm{L}$ compared with only $12 \%$ of women aged 50 to 64 years. Anaemia from any cause (defined as haemoglobin $<125 \mu \mathrm{g} / \mathrm{L}$ ) was present in $23 \%$ of the younger women, suggesting that at least $17 \%$ of these women had iron deficiency without anaemia. No comparable national prevalence data exist in New Zealand to date, but it is likely that many premenopausal New Zealand women 
have mild iron deficiency which may place them at increased risk of developing anaemia if they are exposed to a physiological challenge such as pregnancy, injury or surgery; and which may in itself have adverse health consequences.

Very few studies, however, have examined the characteristics of premenopausal adult women who have low iron stores, and compared them with those of women who are iron sufficient. In the Women's Iron Study, reported here, we have employed a computer-administered food frequency questionnaire to estimate habitual intake, over the past month, of total iron, haem and nonhaem iron, as well as dietary modifiers of iron absorption $^{5}$. In addition, we have collected detailed data on all sources of blood loss, including both the magnitude and duration of menstrual bleeding, the method of contraception used, the frequency of blood donations, and nose bleeds. Finally, we have investigated the importance of both dietary and blood loss factors for risk of mild iron deficiency in a self-selected sample of premenopausal New Zealand women. This is the first comprehensive study of the iron status of a large sample of premenopausal adult women in New Zealand.

\section{Methods}

\section{Participants}

Four hundred and four women aged between 18 and 40 years were recruited from the Greater Dunedin (New Zealand) area, largely via newspaper advertisements and posters. A very small number were recruited through general practitioners. The age group was chosen to maximise the likelihood that all women had both reached menarche and finished growing, and to minimise the likelihood that they had reached menopause ${ }^{6}$.

Ethical approval for this study was granted by the Southern Regional Health Authority Ethics Committee, Otago, New Zealand. Informed written consent was given by all participants.

\section{Data collection and laboratory procedures}

Interested women were eligible to take part in the Women's Iron Study if they were aged 18 to 40 years, were not pregnant or breast-feeding, and were consuming a Western-type diet. Participants completed a General Questionnaire designed to elicit information on sociodemographic status, and dietary patterns, supplement use, recent illness, blood donation, nose bleeds, menstrual blood loss, contraceptive use, cigarette smoking and alcohol drinking. Socio-economic status was determined using the NZSEI scale divided into six classes, as described earlier ${ }^{7}$.

Morning fasting venipuncture blood samples were collected into evacuated tubes (Becton Dickinson, Rutherford, NJ) for biochemical analyses. The stage of the menstrual cycle when the blood test was taken was determined using a record of the date when the blood test was taken; and the participant's estimate of the date when their last period started, how long their menstrual periods last, and the number of days from the beginning of one period to the beginning of the next. The participant was then asked to complete a standard 24-h dietary recall protocol $^{8}$. Height and weight measurements were taken in duplicate using standardised procedures, with participants standing erect in bare feet, and wearing light clothing 9 . A portable stadiometer was used to measure height to the nearest $0.1 \mathrm{~cm}$. Body weight was recorded to the nearest $0.1 \mathrm{~kg}$ using digital scales (SECA 770) from which Quetelet's body mass index was calculated. At a second appointment the Iron Food Frequency Questionnaire and Mood, Activity and Eating Questionnaire were administered. The Mood, Activity and Eating Questionnaire included questions on recent activity (mild: 'time spent on the move'; and vigorous: 'breathing hard or puffing a lot'), and anorexia nervosa and bulimia nervosa (the DSM-IV ${ }^{10}$ ).

Blood samples were refrigerated immediately following collection and analysed within $4 \mathrm{~h}$. Zinc protoporphyrin was measured using a haematofluorimeter (model ProtoFluor-Z) and reagent system (Helena Laboratories) using the manufacturer's controls. The coefficient of variation for this assay was typically 5\%. All other blood sample analyses were conducted at a single laboratory that participates in regular external proficiency testing (RCPA Quality Assurance Program-Endocrine). Serum ferritin was measured by a two site sandwich immunoassay using direct chemiluminescent technology (Chiron Diagnostics Corporation, East Walpole, MA) standardised against World Health Organisation certified reference material (human spleen ferritin: Second International Standard 80/ 578). The serum ferritin assay had a coefficient of variation of $5.7 \%$ at a level of $35 \mu \mathrm{g} / \mathrm{L}$. Haemoglobin was measured by an automated procedure using a Coulter Counter (Beckman Coulter New Zealand Ltd, Auckland). C-reactive protein was measured by nephelometry using a Behring BNA Nephelometer (Dade Behring, Auckland). The C-reactive protein assay had a coefficient of variation of $2.4 \%$.

\section{Measurement of blood loss}

Menstrual blood loss was estimated using a menstrual recall method described and validated earlier in a population of 18- to 29-year-old Dunedin women ${ }^{11}$. The menstrual recall questions asked women to decide how many 'heavy' days they have during a period, and how many 'light' days. They were then asked to state how many pads and/or tampons they use on 'heavy' and 'light' days, and the absorbency level (e.g. 'regular', 'super') of the actual products used. Estimated menstrual loss values generated by the menstrual recall are expressed in Blood Loss Units (BLU).

Participants were asked the following questions on 
blood donation: 'Do you donate blood?', and if so 'When did you last donate blood?' and 'How many times have you donated blood in the past year?'. Participants were asked the following questions on nose bleeds: 'Do you get nose bleeds?', and if so 'How often do you get a nose bleed?' and 'How heavy are your nose bleeds?'. They were also asked whether they used an oral contraceptive pill, an intra-uterine device or depo-provera, and how long they had been using this contraceptive method.

\section{Measurement of dietary intake}

The Iron Food Frequency Questionnaire (Iron FFQ) was used to estimate intake of dietary iron, non-haem iron, haem iron and the modifiers of iron absorption: meat/ fish/poultry, phytate, calcium, vitamin C, tea and coffee ${ }^{12}$ The Iron FFQ is a quantitative computer-administered food frequency questionnaire that asks participants to describe the meals and snacks they have eaten during the past month using 206 foods sorted into 17 food groups. Serving sizes are estimated using multiples and proportions of common standard measures (e.g. cups of coffee, slices of bread), and three-dimensional food models for items such as meat and cheese. As participants complete each individual meal, they are asked to report the exact frequency of consumption for that meal. The food list was developed using sex- and age-specific information from a national 24-h recall to determine the major food sources of each of the dietary components of interest in the diets commonly eaten by New Zealand women aged 18 to 40 years $^{13}$. Selected nutrient intakes were calculated using the New Zealand Food Composition database ${ }^{14}$. Phytate values added to the nutrient database were based on some analysed values and the literature, as described earlier $^{15}$. For composite dishes, phytate content was calculated from recipes published in the New Zealand nutrient database. The Iron FFQ had been validated earlier in a population of 49 Dunedin women aged 19 to 31 years by comparison with 11 days of weighed food records (using the New Zealand Food Composition database $\left.{ }^{14}\right)$. The questionnaire was able to clearly differentiate between low and high intakes of total iron, and iron absorption modifiers. For instance, crossclassification demonstrated that $45 \%$ of meat/fish/poultry intakes were classified in the same quartile by both methods, and no participants were misclassified by the questionnaire into the opposite quartile of weighed diet record intake ${ }^{12}$.

The 24-h recall was used to estimate energy intake (using the New Zealand Food Composition database ${ }^{14}$ ) because the Iron FFQ was not designed to measure macronutrient intake.

\section{Statistical analysis}

SPSS for Macintosh Version 6.1.1 was used to carry out all statistical analyses. Evidence for a difference between groups was tested using the independent groups $t$-test for continuous variables that were normally distributed, the Mann-Whitney $U$ test for those that were not normally distributed, and the Chi-square test for categorical variables. A $P$-value of less than 0.01 was considered to be statistically significant for these analyses because multiple significance tests were being carried out.

Logistic regression analysis ${ }^{16}$ was used to examine the relationship between MID (presence or absence) and a number of characteristics that were of known or suspected biological importance. Continuous independent variables were categorised and treated as nominal variables. Dietary variables were divided into thirds. The remaining continuous variables were converted into meaningful categories. The reference category was the lowest category (or absence) of the characteristic except for variables with another 'natural' reference category (e.g. median duration of menstrual period 4-6 days ${ }^{17}$ ).

Participants with a C-reactive protein (CRP) concentration elevated above $10 \mathrm{mg} / \mathrm{L}^{18}$ were excluded from subsequent analysis $(n=14)$. Individuals with infection are likely to have falsely elevated serum ferritin concentrations ${ }^{19}$ and may therefore be misclassified as having adequate iron status even in the presence of iron deficiency.

Variables for biologically plausible interactions were created, and then added to the full model and tested for statistical significance using the likelihood ratio test ${ }^{16}$.

The ability of the variables in the final logistic regression model to predict the risk of iron deficient erythropoiesis (IDE) was examined using univariate analysis with IDE (presence or absence) as the dichotomous outcome variable.

\section{Results}

Of the original 404 women recruited, 384 (95\%) provided a blood sample. Complete data were provided by 335 participants (87\%) and were included in the logistic regression analysis.

The participants were predominantly Caucasian (95\%) with a median age of 24 years (range: 18 to 40 years). The mean socio-economic status was equivalent to Class 3 (technical workers). The median body mass index was 22.7 (interquartile range: 20.8 to 24.9). Two participants exhibited DSM-IV ${ }^{10}$ defined anorexia nervosa, while none exhibited bulimia nervosa. However, 81 participants (22\%) reported using strict dieting to control their weight in the past year. Eighty-five participants (22\%) avoided eating red meat. The median menstrual blood loss was 35 BLU (range: 2.3 to 292.0 BLU), while 102 participants (27\%) were blood donors (median: 10 months ago) and 77 (20\%) reported having nose bleeds. The oral contraceptive pill was used by 141 participants (37\%). Seven women $(1.8 \%)$ used an intra-uterine device. Ninety-one participants (23.8\%) had taken a multivitamin-mineral supplement in the past year. The amount of supplemental 
Table 1 Characteristics of study participants with and without mild iron deficiency (MID)

\begin{tabular}{|c|c|c|c|}
\hline Characteristic & Participants with MID $(n=87)$ & Participants without MID $(n=297)$ & $P$-value for difference \\
\hline Age (years) & $24.0(20.0,34.0)$ & $24.0(20.0,32.0)$ & 0.90 \\
\hline Body mass index & $21.8(20.4,24.5)$ & $23.0(21.1,25.1)$ & 0.01 \\
\hline Socio-economic status & $3.3(1.6)$ & $3.3(1.6)$ & 0.96 \\
\hline Previous history of MID (\%) & 50.0 & 54.3 & 0.51 \\
\hline Previous history of IDA (\%) & 24.7 & 23.8 & 0.87 \\
\hline Has children (\%) & 21.8 & 22.3 & 0.93 \\
\hline Uses strict dieting to control weight (\%) & 12.8 & 24.1 & 0.02 \\
\hline Recent vigorous activity (\%) & 66.3 & 72.9 & 0.24 \\
\hline Menstrual blood loss (BLU) & $38.0(25.8,60.0)$ & $34.0(22.5,51.8)$ & 0.09 \\
\hline Length of period (days) & $5.2(1.0)$ & $4.9(1.3)$ & 0.02 \\
\hline Donates blood (\%) & 32.2 & 25.0 & 0.18 \\
\hline Has nose bleeds (\%) & 27.6 & 17.9 & 0.05 \\
\hline Uses oral contraceptive pill (\%) & 27.6 & 39.7 & 0.04 \\
\hline Haemoglobin (g/L) & $130.7(6.7)$ & $132.2(8.8)$ & 0.02 \\
\hline Serum ferritin $(\mu \mathrm{g} / \mathrm{L})$ & $15.0(13.0,17.0)$ & $40.0(28.0,64.0)$ & $<0.001$ \\
\hline Elevated C-reactive protein (\%) & 0 & 4.7 & 0.05 \\
\hline Energy (MJ) & $7.6(6.1,9.4)$ & $8.0(6.3,9.8)$ & 0.39 \\
\hline Total iron (mg) & $10.7(8.9,12.4)$ & $10.7(8.5,12.8)$ & 0.42 \\
\hline Haem iron $(\mathrm{mg})$ & $0.7(0.1,1.2)$ & $1.0(0.5,1.4)$ & 0.02 \\
\hline Non-haem iron (mg) & $10.1(8.2,12.3)$ & $9.6(7.7,11.6)$ & 0.14 \\
\hline Meat/fish/poultry (g) & $85.9(61.6)$ & $110.6(66.6)$ & 0.002 \\
\hline Vitamin $C(\mathrm{mg})$ & $105.0(72.7,158.1)$ & $111.8(74.5,163.9)$ & 0.41 \\
\hline Phytate (mg) & $1274.8(755.9,2003.4)$ & $1146.0(674.4,1629.9)$ & 0.06 \\
\hline Calcium (mg) & $725.2(520.9,951.7)$ & $696.9(516.7,901.9)$ & 0.51 \\
\hline Drinks tea (\%) & 56.3 & 51.9 & 0.47 \\
\hline Drinks coffee $(\%)$ & 48.3 & 50.9 & 0.67 \\
\hline Avoids red meat (\%) & 29.9 & 19.9 & 0.05 \\
\hline Current iron supplement (\%) & 19.5 & 17.5 & 0.66 \\
\hline Multivitamin-mineral in past year (\%) & 18.4 & 25.3 & 0.75 \\
\hline
\end{tabular}

Values are expressed as mean (standard deviation), median (interquartile range) or percentage.

iron being taken ranged from $0.06 \mathrm{mg} /$ day (for an infrequently taken multivitamin-mineral supplement) to $105 \mathrm{mg} /$ day (for an iron-only supplement taken daily), with a median intake of elemental iron from supplements of $8.6 \mathrm{mg} /$ day. The majority of the women were nulliparous $(n=299 ; 78 \%)$. The mean parity amongst those women who had children was 2.1.

The median serum ferritin concentration for the population was $32 \mu \mathrm{g} / \mathrm{L}$, with a mean haemoglobin level of $132 \mathrm{~g} / \mathrm{L}$. Eighty-seven participants (23\%) had MID (haemoglobin $\geq 120 \mathrm{~g} / \mathrm{L}$ and serum ferritin $<20 \mu \mathrm{g} / \mathrm{L}$ ), of whom 15 (4\%) had IDE (serum ferritin $<12 \mu \mathrm{g} / \mathrm{L}$ ). Eight participants (2\%) had iron deficiency anaemia (haemoglobin $<120 \mathrm{~g} / \mathrm{L}$ and serum ferritin $<12 \mu \mathrm{g} / \mathrm{L}$ ). An elevated C-reactive protein of greater than $10 \mathrm{mg} / \mathrm{L}$ was measured in 14 participants (4\%). Nine women (2\%) had anaemia without iron deficiency.

Table 1 compares the characteristics of participants with and without mild iron deficiency. Participants with MID (serum ferritin $<20 \mu \mathrm{g} / \mathrm{L}$ in the absence of anaemia (haemoglobin $\geq 120 \mathrm{~g} / \mathrm{L}$ )) had a slightly, but significantly $(P=0.01)$, lower BMI than participants without MID (21.8 and 23.0, respectively) and a significantly lower intake of meat/fish/poultry (86 g vs. $111 \mathrm{~g})(P<0.01)$.

Logistic regression analysis suggested that the following variables were important predictors of MID risk: meat/ fish/poultry intake and menstrual blood loss (Tables 3 and 4), duration of the menstrual period, months since last blood donation, nose bleeds, body mass index, and multivitamin-mineral supplement use in the past year (Table 2). An interaction effect between meat/fish/poultry intake and menstrual blood loss was also apparent in the model (Tables 3 and 4). There was no significant effect of stage of the menstrual cycle ${ }^{20}$ on the results.

Medium and high meat/fish/poultry intakes were associated with decreased risk of MID in individuals with low menstrual blood loss (odds ratios $(\mathrm{OR})=0.22$ and 0.26 ), but not in those with medium or high levels of menstrual loss (Table 3).

Low levels of menstrual blood loss were associated with a highly significant decrease in risk of MID in individuals with medium intakes of meat/fish/poultry $(\mathrm{OR}=0.13)$, and with a marginally significant protective effect for those with high intakes of meat/fish/poultry (Table 4).

Fifteen of the women with mild iron deficiency also showed evidence of IDE (serum ferritin $<12 \mu \mathrm{g} / \mathrm{L}$ and zinc protoporphyrin $\geq 40 \mu \mathrm{mol}$ zinc protoporphyrin $/ \mathrm{mol}$ haem). It was not appropriate to test the ability of the full model to predict IDE because of the small number of women affected. However, univariate logistic regression analysis indicated that recent blood donation $(\mathrm{OR}=5.1$; 
Table 2 Independent risk factors for mild iron deficiency*

\begin{tabular}{|c|c|c|c|c|}
\hline Variable & $n$ & Odds ratio & $95 \% \mathrm{Cl}$ for odds ratio & $P$-value for odds ratio \\
\hline \multicolumn{5}{|l|}{ Duration of menstrual period } \\
\hline$<4$ days & 205 & 0.35 & $0.16-0.77$ & 0.009 \\
\hline 4-6 days & 92 & 1.00 & & \\
\hline$>6$ days & 38 & 0.55 & $0.20-1.48$ & 0.24 \\
\hline \multicolumn{5}{|c|}{ Months since last donated blood } \\
\hline Does not donate blood & 251 & 1.00 & & \\
\hline$>12$ months & 34 & 0.74 & $0.28-1.99$ & 0.56 \\
\hline 4-12 months & 24 & 1.17 & $0.40-3.41$ & 0.77 \\
\hline$<4$ months & 26 & 7.27 & $2.79-18.92$ & $<0.001$ \\
\hline \multicolumn{5}{|l|}{ Has nose bleeds } \\
\hline No & 268 & 1.00 & & \\
\hline Yes & 67 & 2.15 & $1.12-4.11$ & 0.02 \\
\hline \multicolumn{5}{|l|}{ Body mass index } \\
\hline$<20$ & 41 & 2.50 & $1.14-5.50$ & 0.02 \\
\hline $20-25$ & 216 & 1.00 & & \\
\hline$>25$ & 78 & 0.79 & $0.39-1.61$ & 0.51 \\
\hline \multicolumn{5}{|c|}{ Multivitamin/mineral supplement in past year } \\
\hline No & 252 & 1.00 & & \\
\hline Yes & 83 & 0.48 & $0.24-0.98$ & 0.04 \\
\hline
\end{tabular}

* Adjusted for the interaction between menstrual blood loss and meat/fish/poultry intake.

Table 3 The effect of meat/fish/poultry consumption on risk of mild iron deficiency in women with low, medium, or high menstrual blood loss*

\begin{tabular}{lccr}
\hline & Odds ratio & $95 \%$ Cl for odds ratio & $P$-value for odds ratio \\
\hline $\begin{array}{l}\text { Menstrual blood loss <35 BLU } \\
\text { Low meat/fish/poultry intake }\end{array}$ & 1.00 & & \\
Medium meat/fish/poultry intake & 0.22 & $0.09-0.56$ & 03 \\
High meat/fish/poultry intake & 0.26 & $0.10-0.67$ & 0.002 \\
Menstrual blood loss 35-71 BLU & & & 0.005 \\
Low meat/fish/poultry intake & 1.00 & & 51 \\
Medium meat/fish/poultry intake & 0.95 & $0.38-2.38$ & 0.91 \\
High meat/fish/poultry intake & 0.94 & $0.36-2.48$ & 0.90 \\
Menstrual blood loss >71 BLU & & & 47 \\
Low meat/fish/poultry intake & 1.00 & & 41 \\
Medium meat/fish/poultry intake & 1.67 & $0.25-11.07$ & 0.60 \\
High meat/fish/poultry intake & 0.93 & $0.15-5.61$ & 0.93 \\
\hline
\end{tabular}

${ }^{*}$ Adjusted for duration of menstrual period, months since last donated blood, has nose bleeds, body mass index, and multivitamin/mineral supplement use in past year.

Table 4 The influence of menstrual blood loss on risk of mild iron deficiency in women with low, medium, or high consumption of meat/fish/ poultry ${ }^{*}$

\begin{tabular}{|c|c|c|c|c|}
\hline & Odds ratio & $95 \% \mathrm{Cl}$ for odds ratio & $P$-value for odds ratio & $n$ \\
\hline \multicolumn{5}{|l|}{ Meat/fish/poultry low } \\
\hline Menstrual blood loss $<35$ BLU & 1.03 & $0.22-4.68$ & 0.97 & 63 \\
\hline Menstrual blood loss $35-71 \mathrm{BLU}$ & 0.57 & $0.12-2.76$ & 0.49 & 47 \\
\hline Menstrual blood loss $>71 \mathrm{BLU}$ & 1.00 & & & 8 \\
\hline \multicolumn{5}{|l|}{ Meat/fish/poultry medium } \\
\hline Menstrual blood loss <35 BLU & 0.13 & $0.03-0.59$ & 0.008 & 59 \\
\hline Menstrual blood loss 35-71 BLU & 0.32 & $0.08-1.32$ & 0.12 & 49 \\
\hline Menstrual blood loss $>71 \mathrm{BLU}$ & 1.00 & & & 10 \\
\hline \multicolumn{5}{|l|}{ Meat/fish/poultry high } \\
\hline Menstrual blood loss <35 BLU & 0.29 & $0.07-1.11$ & 0.07 & 51 \\
\hline Menstrual blood loss 35-71 BLU & 0.58 & $0.16-2.14$ & 0.41 & 41 \\
\hline Menstrual blood loss >71 BLU & 1.00 & & & 14 \\
\hline
\end{tabular}

* Adjusted for duration of menstrual period, months since last donated blood, has nose bleeds, body mass index, and multivitamin/mineral supplement use in past year. 
$\mathrm{CI}=2.3-774.1) \quad$ and nose bleeds $\quad(\mathrm{OR}=3.0 ; \quad \mathrm{CI}=$ 1.1-146.2) were also significant risk factors for IDE.

Univariate analysis suggested a significant protective effect of oral contraceptive use on risk of MID (OR = $0.58 ; \quad C I=0.34-0.98 ; \quad P=0.04)$. However, once the extent and duration of menstrual bleeding were controlled for, the effect of oral contraceptive pill use disappeared. This indicates that the effect of oral contraceptive pill use on MID was entirely explained by their effect on menstrual blood loss. Similarly, although univariate analysis of haem iron intake $(\mathrm{OR}=0.74 ; \mathrm{CI}=$ $0.55-1.00 ; P=0.05)$, 'avoidance of red meat' $(\mathrm{OR}=1.72$; $\mathrm{CI}=1.00-2.95 ; P=0.05)$, and 'no reported meat $/$ fish/ poultry intake in the Iron FFQ' $(\mathrm{OR}=2.41 ; \mathrm{CI}=$ $1.11-5.22 ; P=0.03)$ suggested a significant increase in risk of MID amongst vegetarians, once the variables in the final model were controlled for, vegetarianism was not associated with MID.

There were some differences between the individuals who were excluded from the final analysis of the risk factors for MID because of incomplete data, or elevated CRP levels, and those who were included. The excluded individuals were older (29 years vs. 26 years). Nevertheless, this is unlikely to have influenced our results because there is no biologically plausible relationship between age and risk of MID, independent of factors such as parity and menstrual blood loss. In addition, the excluded group had a higher meat/fish/poultry intake (134 g vs. $101 \mathrm{~g}$ ), but since they also had higher serum ferritin concentrations $(70 \mu \mathrm{g} / \mathrm{L}$ vs. $38 \mu \mathrm{g} / \mathrm{L}$ ) their inclusion would have increased the likelihood of finding a protective effect of meat/fish/poultry on MID risk.

\section{Discussion}

MID was the focus of the Women's Iron Study because of its suggested association with a number of adverse health consequences $^{1-3}$. There is very little information on the aetiology and consequences of this condition in women. Moreover, the criteria used to define MID have not been well established. In this study we selected a cut-off value for serum ferritin of less than $20 \mu \mathrm{g} / \mathrm{L}$ because impaired endurance performance has been reported in nonanaemic women with serum ferritin concentrations below this cut-off, suggesting that health deficits may be associated with this level of iron deficiency ${ }^{2}$. Further, while Hallberg et al. ${ }^{21}$ chose to use less than $16 \mu \mathrm{g} / \mathrm{L}$ as their cut-off for iron depletion, their data suggest that a cut-off of less than $20 \mu \mathrm{g} / \mathrm{L}$ would result in a higher sensitivity (i.e. $86 \%$ vs. $75 \%$ iron deficient women correctly identified).

Applying these criteria for MID (serum ferritin $<20 \mu \mathrm{g} / \mathrm{L}$; haemoglobin $\geq 120 \mathrm{~g} / \mathrm{L}$ ), $23 \%$ of the women had biochemical evidence of mild iron deficiency. This figure is markedly higher than those reported for premenopausal adult women in most other Western countries for which data on MID (serum ferritin $<20 \mu \mathrm{g} / \mathrm{L}$ ) are available ${ }^{22-24}$, with the exception of Finland (i.e. 30\%) ${ }^{25}$. None of these studies, however, are based on national prevalence data. Our population was a volunteer sample of New Zealand women, $50 \%$ of whom reported a previous history of iron deficiency. Participants were recruited largely by advertisements requesting volunteers for a 'Women's Iron Study'. While it is possible that this attracted a particular group of women volunteers, we believe that they were sufficiently representative of the general population of New Zealand women of this age group. Young New Zealand women in general have a heightened awareness of the importance of iron following ongoing publicity campaigns about iron deficiency both in print media and on television. Moreover, the participants had median intakes of energy $(7.9 \mathrm{MJ})$ and iron $(10.7 \mathrm{mg})$ that were similar to those reported in a nationally representative sample of New Zealand women aged 15 to 44 years studied in 1997 (8.2 MJ and $10.1 \mathrm{mg}$, respectively) ${ }^{26}$.

\section{Aetiological factors associated with risk of MID}

We identified two major aetiological factors as determinants of MID for the women in our study: blood loss and dietary intake, both of which are discussed in detail below.

Three types of blood loss were significant risk factors for MID: recency of blood donation, menstrual blood loss (both extent and duration of menstrual bleeding), and nose bleeds. Very few other studies have demonstrated in a single population the impact of such a comprehensive range of blood loss factors on indices of iron status, and to our knowledge, none has ever investigated the importance of nose bleeds.

Of the three types of blood loss, blood donation resulted in the largest increase in risk of MID, but only amongst women who had donated blood within the past 4 months. The influence of blood donation on risk of MID is likely to be a temporary physiological response to donation. A study by Garry et al. ${ }^{27}$ of postmenopausal women who repeatedly donated blood, suggests that iron loss following a blood donation is initially recouped from iron stores, but repeated donations after the first 3 months result in an increase in iron absorption to meet the additional iron requirement. Premenopausal women blood donors do, however, appear to have lower serum ferritin concentrations than non-donors ${ }^{23,25,28,29}$

Few studies have attempted to investigate the influence of the extent of menstrual blood loss on serum ferritin concentration. Most have used surrogate measures that are more easily assessed, such as menstrual status (e.g. pre- or post-menopause), or the duration or frequency of menstruation ${ }^{30-32}$. Of these studies, one found no association between self-reported 'volume' of menstrual blood loss and serum ferritin concentration ${ }^{30}$ and one found an association with a measure of very heavy menstrual bleeding ('bleeding through pads') ${ }^{32}$. 
Kenneyet $a l .{ }^{31}$ used a 'menstrual index' which included information on the duration of menstrual bleeding and a self-report of its heaviness. Because women's self-reports of the heaviness of their menstrual periods is notoriously inaccurate ${ }^{33}$, these researchers were, in effect, investigating an association between serum ferritin concentration and the duration of menstruation. Our study demonstrates that both the extent and the duration of menstrual blood loss impact on the risk of MID. Our finding that duration of the menstrual period was a risk factor for MID is consistent with that of most other investigators who have examined the influence of menses on serum ferritin concentrations $^{23-25,34-36}$.

Although up to $60 \%$ of people experience one episode of epistaxis (i.e. nose bleeds) in their lifetime, and 6\% will seek medical attention for the condition ${ }^{37}$, no other study to our knowledge has investigated the influence of nose bleeds on the risk of iron deficiency. Nose bleeds (experienced by $20 \%$ of this population) doubled the risk of MID in this study.

Unlike blood loss, only one dietary factor-intake of meat/fish/poultry - was a significant predictor of risk for MID in this New Zealand study. This finding is not surprising. The protective effect of flesh foods can be explained by their high content of bioavailable haem iron $^{38}$, as well as the concomitant enhancing effect of flesh foods on non-haem iron absorption ${ }^{39}$. Further, of all the dietary variables known to modify the bioavailability of iron, intakes of meat, poultry and fish have been most consistently associated with serum ferritin concentration $^{22,29,30,32,36,40,41}$. Intakes of meat/fish/poultry and haem iron in the diets of these New Zealand women were low, whereas intakes of total dietary iron, vitamin C and calcium were moderate compared with those reported for premenopausal women in other countries.

An interaction between flesh food intake and menstrual blood loss is reported here in which only those with a low menstrual blood loss were able to decrease their risk of MID with higher intakes of flesh foods. This is presumably explained by the less negative iron balance of individuals with a lower menstrual iron loss.

Interestingly, vegetarianism per se was not a significant predictor of MID risk in our population, although being in the lowest third of meat/fish/poultry intakes ( $<79 \mathrm{~g} /$ day $)$ did increase risk. Donovan and Gibson ${ }^{42}$ reported that semi-vegetarian adolescent females who excluded red meat had poorer iron intakes and a higher prevalence of low iron stores ${ }^{15}$ than either vegetarians or omnivores, suggesting that a poorly planned diet rather than vegetarianism itself may increase risk of iron deficiency.

The absence of an effect of total iron intake on risk of MID reported in this study is not surprising, in view of the range of dietary components known to modify the bioavailability of iron. The two major studies that have reported an association between total iron intake and serum ferritin concentrations have included both men and women and covered a wide age range, maximising the range of intakes and therefore the likelihood of finding an association $^{40,43}$. Moreover, with the exception of flesh foods discussed above, consumption of none of the other dietary components known to enhance (e.g. vitamin C) or inhibit (e.g. calcium, phytate, tea, coffee) iron absorption and measured by our computerised food frequency questionnaire were implicated in the risk for MID. Several other researchers have failed to demonstrate a positive relationship between vitamin $\mathrm{C}$ intake and serum ferritin concentration $^{30,34,43}$ possibly because the biological availability of dietary iron depends on the amount of vitamin $\mathrm{C}$ consumed at mealtimes rather than the total daily intake, as noted by Razagui et $a l^{35}$.

A negative association between calcium or dairy product intake and serum ferritin levels has been found in many ${ }^{24,31,32,34,36,40,43}$ but not all $^{22}$ cross-sectional studies. This finding has not been confirmed in longterm intervention studies ${ }^{44-47}$. In a single meal study, the inhibitory effects of calcium on iron absorption reportedly reached a maximum level at intakes of $300 \mathrm{mg}^{48}$. Participants in our study had a mean calcium intake of more than $700 \mathrm{mg} /$ day. If the majority of this calcium intake was at one or two meals, and there is an inhibitory effect of calcium on iron absorption, then most individuals would have been experiencing the maximum inhibitory effect of calcium. Therefore, any gradient in calcium intakes would not result in gradients in iron absorption. Another explanation may be that calcium intakes in the cross-sectional studies may have reflected the consumption of dairy products (such as cheese), substituted for foods with a higher iron content (such as meat), rather than any inhibitory effect of calcium per se on iron absorption.

In order to investigate whether individuals with traditionally defined pre-anaemic IDE exhibited similar aetiological characteristics to our mildly iron deficient group, we applied the Serum Ferritin model for determining iron deficiency used in NHANES II ${ }^{49}$. There were only 15 women in our population with IDE defined by abnormal serum ferritin $(<12 \mu \mathrm{g} / \mathrm{L})$ and zinc protoporphyrin ( $\geq 40 \mu \mathrm{mol}$ zinc protoporphyrin/mol haem). Although the very wide confidence intervals indicate a lack of precision because there were so few cases in the analysis, two risk factors for MID were also significant risk factors for IDE: blood donation $(\mathrm{OR}=5.1$; $\mathrm{CI}=$ 2.3-774.1) and nose bleeds (OR = 3.0; $\mathrm{CI}=1.1-146.2)$.

\section{Possible strategies for reducing risk of MID in young adult women}

Our study is the first to demonstrate an interaction between MID and two of the risk factors: intake of flesh foods, and menstrual blood loss. This interaction is important because it emphasises that risk for MID in this study group is potentially modifiable. Individuals with a low intake of meat/fish/poultry may be able to decrease 
their risk of MID by increasing their intake of flesh foods provided their menstrual blood loss is low. It is important to note that the New Zealand women in our study did not consume large amounts of meat (in comparison with the intakes reported for French women by Galán et al. ${ }^{34}$ and Soustre et $a l .{ }^{36}$, for instance). Therefore, it is possible that higher intakes of meat/fish/poultry than those represented in our population may be sufficient to overcome higher levels of menstrual blood loss.

Women with moderate to high intakes of flesh foods (as defined by our population) and high levels of menstrual loss may choose to reduce their risk of MID by changing their method of contraception to reduce their menstrual blood loss. Oral contraceptive use decreases menstrual blood loss by about 50\% ${ }^{50}$, while traditional intra-uterine devices (IUDs) may increase menstrual bleeding by as much as $100 \%^{51}$. These differences in the magnitude of menstrual blood loss are reflected in differences in serum ferritin concentration ${ }^{23}$. Women with very heavy menstrual blood losses may need more intensive management to decrease their blood loss ${ }^{52}$.

Menstruating women who donate blood may find it prudent to have their iron stores monitored, as well as their haemoglobin levels. Moreover, such women should aim to develop an individualised programme of blood donation and iron supplementation with their health professional that enables them to maintain their iron stores. Likewise, nose bleeds should be considered as an aetiological factor in iron deficiency, and iron status should be investigated in those who experience them.

Use of a vitamin and mineral supplement in the past year was shown to reduce the risk of MID in this study, despite their irregular use and the low levels of iron (3$15 \mathrm{mg}$ ) contained in most of the multivitamin-mineral supplements sold to the adult female population in New Zealand. However, the protective effect of multivitaminmineral supplement consumption was apparently not a marker for a general interest in health, motivated possibly by an earlier diagnosis of iron deficiency and/or healthier eating patterns. A previous medical diagnosis of low iron status did not help predict the risk of MID in this study. Moreover, supplement use remained significant even after meat/fish/poultry intake was controlled for in the model, emphasising a role for vitamin and mineral supplement usage in the prevention of MID.

The association noted here between a low BMI and increased risk of MID is yet another reason to discourage young adult women from pursuing the current underweight ideal. This relationship was not due to confounding by vegetarians who tend to be leaner than omnivores $^{53}$, with a lower BMI and a greater risk of MID. When variables such as self-defined vegetarianism, avoidance of red meat, and absence of meat, fish or poultry consumption during the previous month in the IFFQ, were added separately to the logistic regression model they did not alter the model's ability to predict
MID, or alter the contribution of BMI to the model (data not presented). Women with a low BMI (i.e. <20) did not have a significantly lower iron or meat/fish/poultry intake than individuals with a BMI of 20 or more (data not presented).

\section{Conclusions}

In summary, our results demonstrate that the risk of MID in a single population of premenopausal adult New Zealand women is associated with three types of blood loss: menstrual blood loss, recent blood donation and nose bleeds; but only one dietary variable-intake of meat/fish/poultry. Further, the interaction between risk of MID and intake of flesh foods and menstrual blood loss noted here emphasises that young adult women with low menstrual blood loss may be able to decrease their risk of MID by increasing their meat/fish/poultry intake, while those with a higher menstrual blood loss may be able to decrease their risk by decreasing their menstrual blood loss, perhaps by changing their method of contraception. Premenopausal women who choose to donate blood or who have nose bleeds should monitor their iron stores regularly and use iron supplements when necessary. Women with a low BMI should be encouraged to reach the healthy BMI range of $20-25$ by following a healthy low-fat diet.

\section{Acknowledgements}

This research was supported (in part) by a grant from the Health Research Council of New Zealand. We would also like to thank Southern Community Laboratories Ltd for providing us with interview rooms, and advice on the interpretation of individual haematology results; Mother Earth Ltd and Frucor Beverages Ltd for providing the participants with muesli bars and fruit juice following their blood tests; Rebecca Meikle who conducted the interviews; Nick Prosser who collected the height and weight information; Michael Hoffmeister and Philippa Mann who entered the questionnaire data; and the participants who took part in the study. A.-L. M. Heath was supported (in part) by an award from the Health Research Council of New Zealand.

\section{References}

1 Bruner AB, Joffe A, Duggan AK, Casella JF, Brandt J. Randomised study of cognitive effects of iron supplementation in non-anaemic iron-deficient adolescent girls. Lancet 1996; 348: 992-6.

2 Rowland TW, Deisroth MB, Green GM, Kelleher JF. The effect of iron therapy on the exercise capacity of nonanemic iron-deficient adolescent runners. Am. J. Dis. Child. 1988; 142: 165-9.

3 Zhu YI, Haas JD. Iron depletion without anemia and physical performance in young women. Am. J. Clin. Nutr. 1997; 66: $334-41$ 
4 Gregory J, Foster K, Tyler H, Wiseman M. The Dietary and Nutritional Survey of British Adults. London: HMSO, 1990.

5 Allen LH, Ahluwalia N. Improving Iron Status through Diet: The Application of Knowledge Concerning Dietary Iron Bioavailability in Human Populations. Arlington, VA: John Snow, Inc./OMNI Project, 1997.

6 Milman N, Kirchhoff M, Jørgensen T. Iron status markers, serum ferritin and hemoglobin in 1359 Danish women in relation to menstruation, hormonal contraception, parity and postmenopausal hormone treatment. Ann. Hematol. 1992; 65: $96-102$.

7 Davis P, McLeod K, Ransom M, Ongley P. The New Zealand Socioeconomic Index of Occupational Status (NZSEI). Wellington, New Zealand: Statistics New Zealand, 1997.

8 Gibson RS. Nutritional Assessment: A Laboratory Manual. New York: Oxford University Press, 1993.

9 Lohman TG, Roche AF, Martorell R. eds. Anthropometric Standardization Manual. Champagne, IL: Human Kinetic Books, 1988.

10 American Psychiatric Association. Diagnostic and Statistical Manual of Mental Disorders. 4th edition. Washington, DC: American Psychiatric Association, 1994.

11 Heath A-LM, Skeaff CM, Gibson RS. Validation of a questionnaire method for estimating extent of menstrual blood loss in young adult women. J. Trace Elements Med. Biol. 1998; 12: 231-5.

12 Heath A-LM, Skeaff CM, Gibson RS. The relative validity of a computerized food frequency questionnaire for estimating intake of dietary iron and its absorption modifiers. Eur. J. Clin. Nutr. 2000; 54: 592-9.

13 LINZ Activity \& Health Research Unit. Twenty Four Hour Diet Recall: Nutrient Analysis Based on 1992 DSIR Database, April 1992. Dunedin, New Zealand: LINZ Activity \& Health Research Unit, University of Otago, 1992.

14 Burlingame BA, Milligan GC, Quigley RJ, Spriggs T. FOODfiles Manual. Wellington, NZ: New Zealand Institute for Crop and Food Research Ltd, 1995.

15 Donovan UM, Gibson RS. Iron and zinc status of young women aged 14 to 19 years consuming vegetarian and omnivorous diets. J. Am. Coll. Nutr. 1995; 14: 463-72.

16 Hosmer DW, Lemeshow S. Applied Logistic Regression. New York: John Wiley \& Sons, 1989.

17 Rybo G. Menstrual blood loss in relation to parity and menstrual pattern. Acta Obstet. Gynecol. Scand. 1966; 45: $25-45$.

18 Hobbs FDR, Kenkre JE, Carter YH, Thorpe GH, Holder RL. Reliability and feasibility of a near patient test for C-reactive protein in primary care. Br. J. Gen. Prac. 1996; 46: 395-400.

19 Hulthén L, Lindstedt G, Lundberg P-A, Hallberg L. Effect of a mild infection on serum ferritin concentration-clinical and epidemiological implications. Eur. J. Clin. Nutr. 1998; 52: 376-9.

20 Kim I, Yetley EA, Calvo MS. Variations in iron-status measures during the menstrual cycle. Am. J. Clin. Nutr. 1993; 58 705-9.

21 Hallberg L, Bengtsson C, Lapidus L, Lindstedt G, Lundberg PA, Hultén L. Screening for iron deficiency: an analysis based on bone marrow examinations and serum ferritin determinations in a population sample of women. Br. J. Haematol. 1993; 85: 787-98.

22 Brussaard JH, Brants HAM, Bouman M, Löwik MRH. Iron intake and iron status among adults in the Netherlands. Eur.J Clin. Nutr. 1997; 51: S51-8.

23 Milman N, Rosdahl N, Lyhne N, Jørgensen T, Graudal N. Iron status in Danish women aged 35-65 years: relation to menstruation and method of contraception. Acta Obstet. Gynecol. Scand. 1993; 72: 601-5.

24 Rangan AM, Aitken I, Blight GD, Binns CW. Factors affecting iron status in 15-30 year old female students. Asia Pacific J. Clin. Nutr. 1997; 6: 291-5.
25 Fogelholm M, Alopaeus K, Silvennoinen T, Teirilä J. Factors affecting iron status in non-pregnant women from urban South Finland. Eur. J. Clin. Nutr. 1993; 47: 567-74.

26 Russell D, Parnell W, Wilson N. NZ Food: NZ People: Key Results of the 1997 National Nutrition Survey. Wellington, NZ: Ministry of Health, 1999.

27 Garry PJ, Koehler KM, Simon TL. Iron stores and iron absorption: effects of repeated blood donations. Am. J. Clin. Nutr. 1995; 62: 611-20.

28 Finch CA, Cook JD, Labbe RF, Culala M. Effect of blood donation on iron stores as evaluated by serum ferritin. Blood 1977; 50: 441-7.

29 Leggett BA, Brown NN, Bryant SJ, Duplock L, Powell LW, Halliday JW. Factors affecting the concentrations of ferritin in serum in a healthy Australian population. Clin. Chem. 1990; 36: $1350-5$.

30 Bairati I, Herbeth B, Spyckerelle Y, et al. Dietary intake and other determinants of iron and folate status in female adolescents. J. Clin. Bioc. Nutr. 1989; 7: 143-51.

31 Kenney MA. Factors related to iron nutrition of adolescent females. Nutr. Res. 1985; 5: 157-66.

32 Yokoi K, Alcock NW, Sandstead HH. Iron and zinc nutriture of premenopausal women: associations of diet with serum ferritin and plasma zinc disappearance and of serum ferritin with plasma zinc and plasma zinc disappearance. J. Lab. Clin. Med. 1994; 124: 852-61.

33 Hallberg L, Högdahl A-M, Nilsson L, Rybo G. Menstrual blood loss-a population study: variation at different ages and attempts to define normality. Acta Obstet. Gynecol. Scand. 1966; 45: 320-51.

34 Galán S, Hercberg S, Soustre Y, Dop MC, Dupin H. Factors affecting iron stores in French female students. Hum. Nutr.: Clin. Nutr. 1985; 39C: 279-87.

35 Razagui IB, Barlow PJ, Izmeth MGA, Taylor KDA. Iron status in a group of long-stay mentally handicapped menstruating women: some dietary considerations. Eur. J. Clin. Nutr. 1991; 45: 331-40.

36 Soustre Y, Dop MC, Galan P, Hercberg S. Dietary determinants of the iron status in menstruating women. Int. J. Vit. Nutr. Res. 1986; 56: 281-6.

37 Small M, Murray J, Maran A. A study of patients with epistaxis requiring admission to hospital. Health Bull. 1982; 40: 24.

38 Acosta A, Amar M, Cornbluth-Szarfarc SC, et al. Iron absorption from typical Latin American diets. Am. J. Clin. Nutr. 1984; 39: 953-62.

39 Hallberg L, Rossander L. Improvement of iron nutrition in developing countries: comparison of adding meat, soy protein, ascorbic acid, citric acid, and ferrous sulphate on iron absorption from a simple Latin American-type of meal. Am. J. Clin. Nutr. 1984; 39: 577-83.

40 Galan P, Yoon H-C, Preziosi P, et al. Determining factors in the iron status of adult women in the SU.VI.MAX study. Eur. J. Clin. Nutr. 1998; 52: 383-8.

41 Worthington-Roberts BS, Breskin MW, Monsen ER. Iron status of premenopausal women in a university community and its relationship to habitual dietary sources of protein. Am. J. Clin. Nutr. 1988; 47: 275-9.

42 Donovan UM, Gibson RS. Dietary intakes of adolescent females consuming vegetarian, semi-vegetarian, and omnivorous diets. J. Adol. Health 1996; 18: 292-300.

43 Preziosi P, Hercberg S, Galan P, Devanlay M, Cherouvrier F, Dupin H. Iron status of a healthy French population: factors determining biochemical markers. Ann. Nutr. Metab. 1994; 38: $192-202$.

44 Ilich-Ernst JZ, McKenna AA, Badenhop NE, et al. Iron status, menarche, and calcium supplementation in adolescent girls. Am.J. Clin. Nutr. 1998; 68: 880-7.

45 Minihane AM, Fairweather-Tait SJ. Effect of calcium supplementation on daily nonheme-iron absorption and long-term iron status. Am. J. Clin. Nutr. 1998; 68: 96-102. 
46 Reddy MB, Cook JD. Effect of calcium intake on nonhemeiron absorption from a complete diet. Am.J. Clin. Nutr. 1997; 65: $1820-5$.

47 Sokoll LJ, Dawson-Hughes B. Calcium supplementation and plasma ferritin concentrations in premenopausal women. Am.J. Clin. Nutr. 1992; 56: 1045-8.

48 Hallberg L, Brune M, Erlandsson M, Sandberg A-S, Rossander-Hultén L. Calcium: effect of different amounts on nonheme- and heme-iron absorption in humans. Am. J. Clin. Nutr. 1991; 53: 112-19.

49 Johnson MA. Iron: nutrition monitoring and nutrition status assessment. J. Nutr. 1990; 120: 1486-91.
50 Callard GV, Litofsky FS, DeMerre LJ. Menstruation in women with normal or artificially controlled cycles. Fert. Steril. 1966; 17: 684-8.

51 Guttorm E. Menstrual bleeding with intrauterine contraceptive devices. Acta Obstet. Gynecol. Scand. 1971; 50 9-16.

52 Wood CE. Menorrhagia: a clinical update. Med.J. Aust. 1996; 165: $510-14$.

53 Dwyer JT. Health aspects of vegetarian diets. Am. J. Clin. Nutr. 1988; 48: 712-38. 